sorti per iniziativa privata e pubblica. Questi nella nostra regione si affermano soprattutto in forma di abitati rurali. Le istituzioni assistenziali, terapeutiche o ricreative, elementi da centro di cura, sono pure rappresentate. Mentre le colonie di vacanza vi si notano soltanto da un paio di decenni, le strutture religiose del Malcantone sono radicate nei secoli lontani: la ricchezza degli edifici per il culto non è trascurabile nemmeno sotto l'aspetto qualitativo, e spesso dà tono artistico alla regione.

Prima che siano per sempre perduti, occorre salvare alla nostra Patria i minacciati molteplici valori naturali e culturali del Malcantone. La limitazione delle cessioni territoriali agli stranieri, la costituzione di aziende medie razionali, lo sviluppo di un ambiente curativo autonomo, e un incremento professionale nelle industrie della zona alluvionale potrebbero dimostrarsi risolutivi.

\title{
WOHNHÖHLEN IN SÜDITALIEN
}

\author{
Karl Suter
}

Mit vier Abbildungen

An vereinzelten Orten Süditaliens lebt immer noch ein Teil der Bevölkerung in Wohnhöhlen, so zum Beispiel in dem ungefähr 30000 Einwohner zählenden Matera, einem Provinzhauptort der Basilikata. Seine Wohnhöhlen bilden im östlichen Teil der Stadt, im besondern in den Hängen des Sasso Barisano und des Sasso Caveoso, die gegen die zirka $150 \mathrm{~m}$ tief eingeschnittene Gravina di Matera absteigen, ein großes, unter dem Namen Sassi bekanntes Quartier. Da liegen Hunderte von Felswohnungen nebenund übereinander; es müssen im ganzen gegen 20 Stockwerke sein. Wohnhöhlen des gleichen Typs - des Hangtyps - kommen auch andernorts in Europa vor, beispielsweise

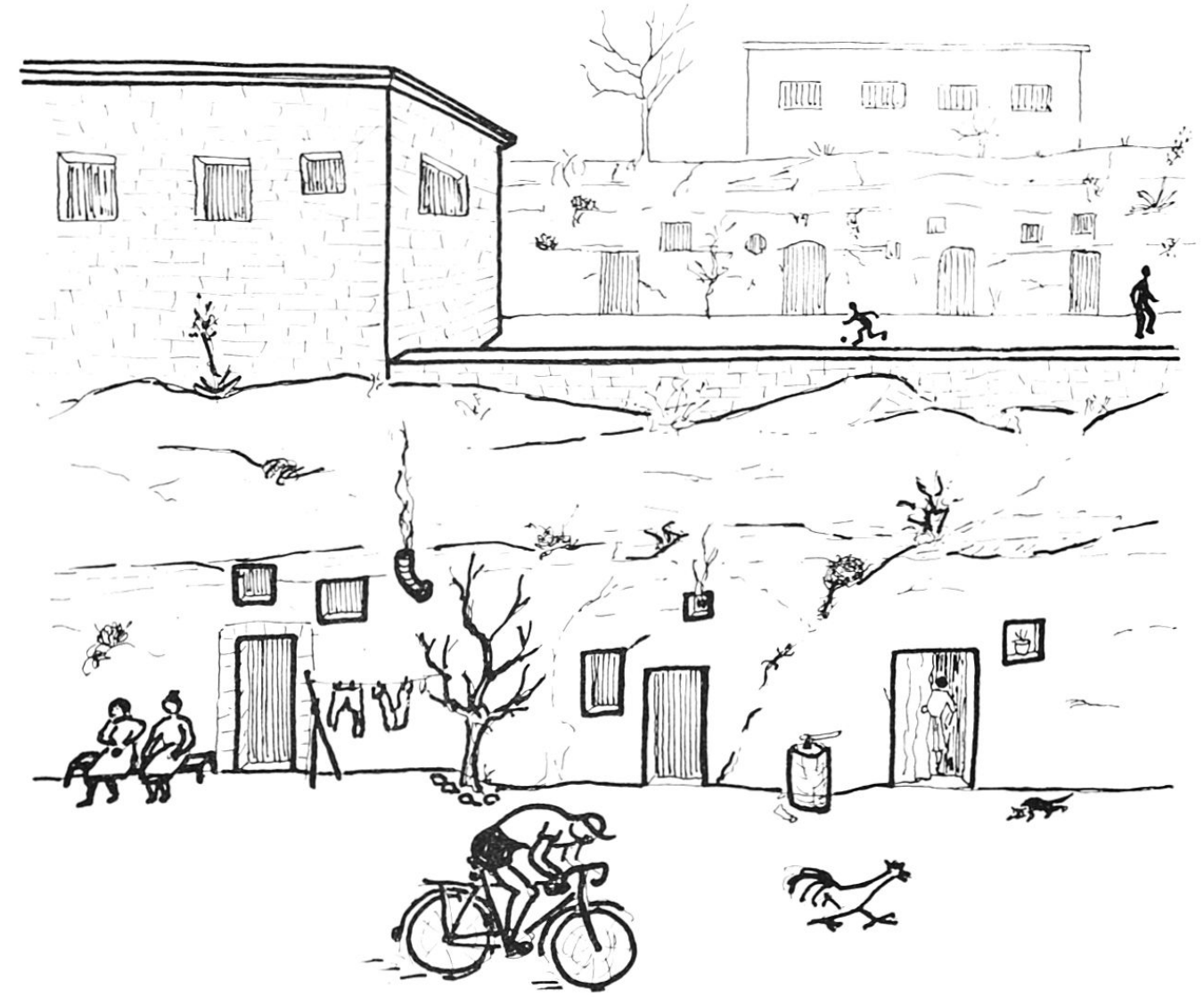

Abb. 1: Matera. Häuser und Grotten im Quartier der Sassi 

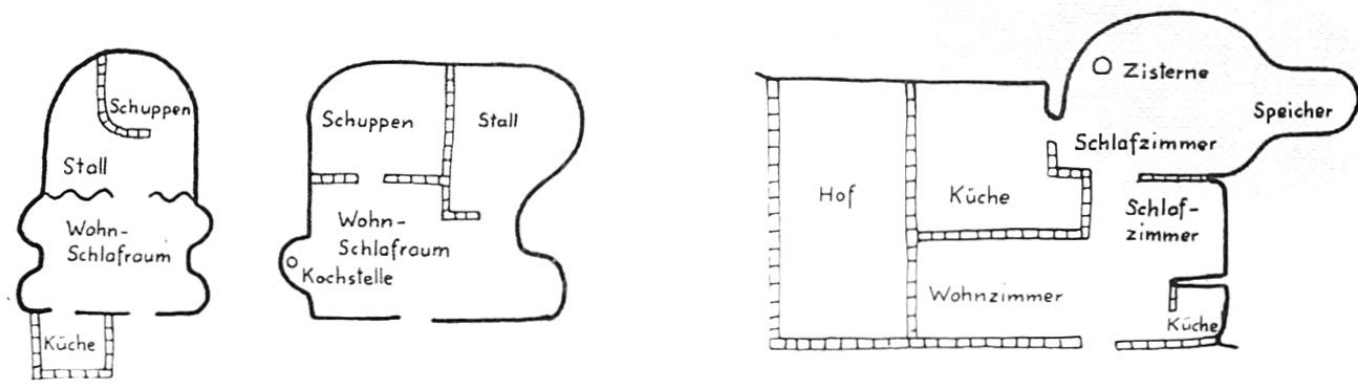

Abb. 2: Matera. Grundrisse von Grotten. Abb. 4: Massafra. Grundriß einer Höhlenwohnung mit Vorbauten

in der Bergstadt Chinchilla der Mancha Spaniens. Wie O. Jessex ${ }^{1}$ (S. 210) berichtet, liegen sie dort ebenfalls terrassenartig übereinander. Während in Chinchilla diese Anordnung aber durch die geologischen Verhältnisse bedingt ist, das heißt durch den Umstand, daß härtere, waagrecht verlaufende Gesteinsschichten mit weichen wechsellagern - die Höhlen sind im weichen Material zwischen den harten Kalkbänken ausgehoben -, ist das in Matera nicht der Fall. Das fast durchwegs gleich beschaffene Gestein, ein gut bearbeitbarer und doch solider Kalktuff, erlaubte den Grottenbau an beliebiger Stelle. Daß dennoch eine stockwerkartige Anordnung zustande kam, geht auf das Bestreben zurück, die Talhänge möglichst gut auszunützen und ein zweckmäßiges Gassennetz zu erstellen. Die einen Gassen ziehen, den Grottenreihen folgend, mehr oder weniger horizontal dahin, die andern aber steigen, die einzelnen Stockwerke miteinander verbindend, von der Hochfläche gegen das Schluchttal nieder, häufig sogar in Form von Treppengassen. In das Gassensystem sind da und dort kleinere Plätze eingeschaltet.

Überblickt man die Sassi von einer exponierten Stelle, zum Beispiel vom Dom aus, so glaubt man ein gewöhnliches, aus lauter Häusern bestehendes, amphitheatralisch sich aufbauendes Quartier vor sich zu haben. Häuser sind in der Tat auch in großer Zahl vorhanden. Sie erheben sich oft über den Grotten, gehören aber nicht zu diesen: Grotten und Häuser werden also von verschiedenen Familien bewohnt. Auf der einen Seite der waagrecht dahinziehenden Gassen geht es in Grotten hinein, auf der andern Seite in einfache, meist zweigeschossige Wohnhäuser (Abb. 1). Vor vereinzelten Grotten liegt eine aus Mauerwerk erstellte Küche mit Kamin. Das verstärkt den Eindruck, wonach die Sassi eine reine Häusersiedlung darstellen, während jedoch die Anzahl der Grotten größer als die der Häuser ist.

Die Wohnhöhlen haben im großen ganzen ein gleichartiges Aussehen. Sie bestehen -wohl durchwegs - bloß aus einem einzigen Raum, indes aus einem auffallend großen. Er ist nicht selten $30 \mathrm{~m}$ lang, $10-20 \mathrm{~m}$ breit und 6-7 $\mathrm{m}$ hoch. Er ist in der Regel durch einen Vorhang, eine Holzwand oder eine Mauer in zwei Abschnitte geteilt (Abb.2). Der vordere, gewöhnlich etwas größere Abschnitt stellt ein Tonnengewölbe dar, hat einen mit Fliesen bedeckten Boden, ist sauber geweißt und wird von der Familie als Wohn- und Schlafraum benützt. Er ist einfach möbliert; neben Betten, Tisch und Stühlen weist er Kästen und Kommoden auf, die in Wandausbuchtungen stehen; dazu kommen Nippsachen, Geschirr usw. in verschiedenen kleinen Wandnischen. Eine besondere Ausbuchtung, und zwar gegen die Gasse hin, dient als Herdstelle; sie besitzt ein die Grottendecke oder Grottenwand durchstoßendes Kamin. Der hintere Abschnitt aber - der größere Teil der Sassi-Bevölkerung treibt Landwirtschaft - stellt den Stall für die Trag- und Reittiere (Esel, Maultier, Pferd) dar. Dort findet sich gewöhnlich noch ein weiteres Abteil, wo Viehfutter, namentlich Stroh, aufbewahrt wird, oft auch noch Gartengeräte, Behälter für Olivenöl oder große, mit Korn angefüllte Kästen. Der

1 O. Jessen: La Mancha. Mitteilungen der Geographischen Gesellschaft in Hamburg 1930: Über Wohnhöhlen. S. 207-2I3. 
Stallboden besteht aus festgestampfter Erde. Die Eingänge in die Grotten sind, da sie ja auch Tieren Durchlaß geben müssen, groß. Über ihnen finden sich ein oder zwei aus dem Fels gehauene Fensteröffnungen. Aborte fehlen; sie werden durch passende Stellen im Freien ersetzt.

Das Wohnen in Grotten wird in Matera heute als unzeitgemäß empfunden. Die Sassi stehen im Ruf, feucht und darum ungesund zu sein. Die Feuchtigkeit rührt vor allem vom Stalle her. Sie ist so groß, daß eiserne Gegenstände rasch rosten und das Holz der Möbel mit der Zeit aus den Fugen geht. Auch erscheint die Grotte im Sommer als zu kühl; der sommerliche Temperaturunterschied zwischen derselben und dem Freien wird oft als groß empfunden. Die Leute klagen über rheumatische Beschwerden, die sie auf das Wohnen in der feuchten, kühlen Grotte zurückführen. Lästig ist ferner der daselbst verbreitete Stallgeruch. Auch kann der Wohn- und Schlafraum wegen des Durchgangs der Tiere nicht genügend sauber gehalten werden.

Diese Grotten sollen aufgegeben werden. Eine $Z_{\text {wangsumsiedlung ist im Gange. Sie }}$ erfolgt in die neuen Dörfer, wie Borgo Venusio, und in die auf der hügeligen Hochfläche im Umkreis des Piano - so heißt der obere, schon längst aus Häusern bestehende Stadtteil gegen den Bahnhof hin - gelegenen neuen Quartiere, wie Serra Venerdi, Spine Bianca, La Nera, La Martella und andere. Diese Quartiere machen einen durchaus städtischen Eindruck, und nichts deutet darauf hin, daß sie von Bauern bewohnt werden. Da Viehställe fehlen, können keine Reittiere mehr gehalten werden; die bereits umgesiedelten Bauern müssen darum den Acker zu Fuß oder mit dem Fahrrad aufsuchen. Nicht wenige haben zu Fuß täglich einen Hin- und Rückweg von ein bis zwei Stunden zurückzulegen. Trotz diesem enormen Zeit- und Kraftverschleiß kehren sie täglich nach Matera zurück und nächtigen nur ausnahmsweise, etwa zu den Erntezeiten, in Feldhütten. Es würde naheliegen, sie auf ihren Grundstücken anzusiedeln. Das kommt aber nicht in Frage; denn die Leute wollen nicht in Einzelhöfen leben, sondern nur in enger Dorf-, Quartier- oder Stadtgemeinschaft. Sie wollen einander sehen und am abendlichen Spaziergang stadtauf, stadtab - dem sogenannten Corso - teilnehmen.

Heute sind nahezu zwei Drittel der über 8000 Sassi-Bewohner, das heißt der ungefähr 1650 Familien, umgesiedelt. Durchwandert man die Sassi, sieht man zahlreiche aufgegebene Grotten. Ihre Eingänge und Fensteröffnungen sind zugemauert. Nur so läßt sich verhindern, daß die ausgezogenen Familien wieder zurückkehren oder sich andere darin einnisten. Die Umsiedlung fällt den Leuten aus verschiedenen Gründen schwer. Erstens wegen der Kosten; das Wohnen in der Grotte ist unvergleichlich viel billiger als im Haus, wo die Miete ein Mehrfaches beträgt. Zweitens haben die Leute Mühe, sich an die neue Wohnform zu gewöhnen. Sie fühlen sich in den kleinen Wohnungen eingeengt. Drittens klagen sie über Wind und Kälte, denen die Häuser auf der Hochfläche, besonders im Winterhalbjahr, im Gegensatz zu den windgeschützten Grotten ausgesetzt sind. Viertens haben sie keinen Stall mehr.

In Wohnhöhlen des Hangtypus lebt heute noch ein Teil der Bevölkerung von Massafra, einer rund 20000 Einwohner zählenden Stadt Apuliens (Provinz Tarent). Wie Matera liegt auch sie auf einem breiten Plateau aus Kalktuff, das von Schluchttälern zerschnitten wird. Zwei davon, die Gravina della Madonna della Scala und die Gravina di San Marco, welche beide nach Süden in die anschließende Küstenebene von Tarent ausmünden, haben ihre Anlage und räumliche Entwicklung maßgebend mitbestimmt. Die Gravina di San Marco teilt sie in zwei Abschnitte: in die schachbrettartig gegliederte Neustadt und in die von winkligen, engen Gassen durchzogene Altstadt. Diese erfreute sich einer guten Verteidigungslage, wird sie doch auch noch im Westen von einem Schluchttal, nämlich der Madonna della Scala, begrenzt.

Die Schluchthänge sind von mediterranen Gewächsen, wie Pinien, Myrten, Johannisbrotbäumen, bedeckt. Zwischen diesen schauen als schwarze Löcher die Eingänge in Höhlen hervor. Doch nur noch ein Teil der Höhlen wird bewohnt, wie etwa jene am rechten Hang der Gravina di San Marco unterhalb des Kastells. Auch in Massafra lie- 


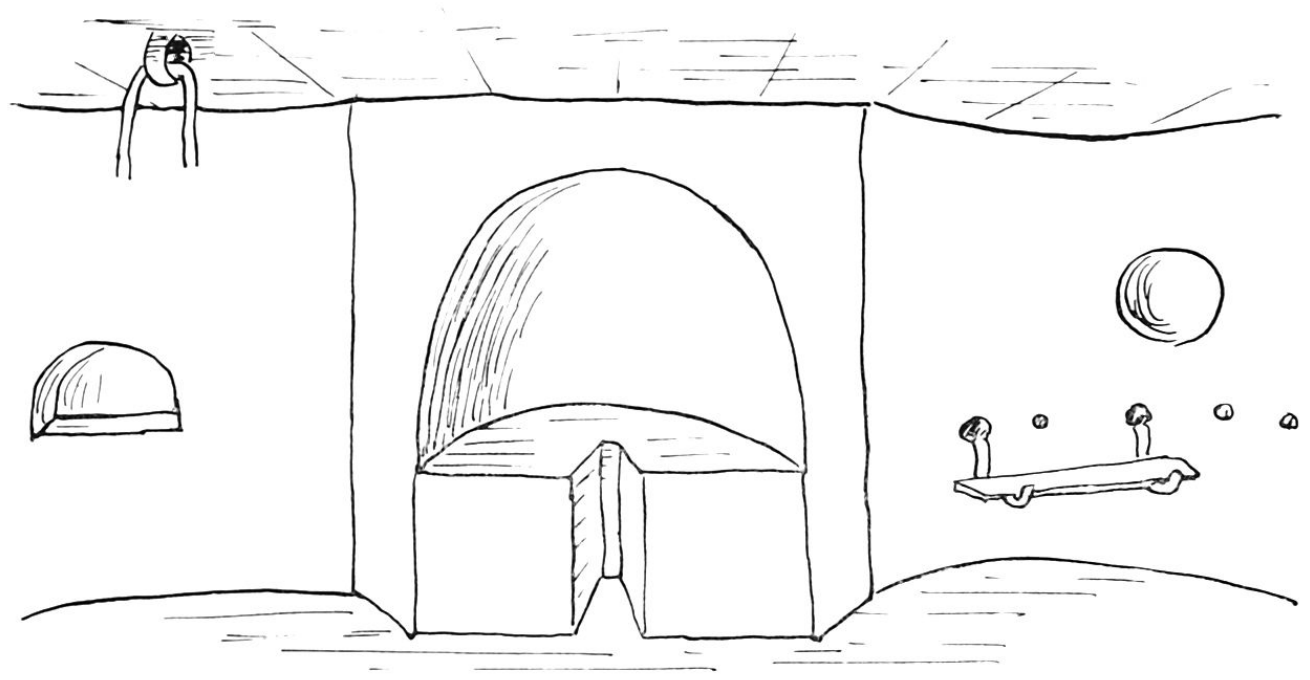

Abb. 3: Massafra. Beispiel einer alten, in der Gravina della Madonna della Scala gelegenen Grotte

gen sie neben- und übereinander, allerdings in meist nur drei oder vier Stockwerken, die durch relativ dünne $Z$ wischenschichten - oft von bloß knapp einem Meter - voneinander getrennt werden. $\mathrm{Zu}$ den höher gelegenen Grotten führen steile Pfade oder, wie das besonders schön in der Gravina della Madonna della Scala zu sehen ist, im Fels ausgehauene Stiegen.

Von ganz besonderem Interesse ist das seit langem unbewohnte Höhlengebiet der Gravina della Madonna della Scala, auf deren Grund die Kirche gleichen Namens steht. Hier kommen Höhlen vor, die jahrhundertealt sein dürften und sich durch besondere Merkmale auszeichnen. Schon in ihren Grundrissen - rundlich bis polygonal sind sie recht verschieden. Daß die meisten bewohnt waren, verraten die in der Regel neben den Eingängen gelegenen Kochstellen mit Rauchabzugsloch (Durchmesser 40 bis $60 \mathrm{~cm}$ ) und mit faustgroßen Wandeintiefungen für die Aufnahme von beim Kochen benötigten Zutaten. Den eigenartigsten Bauteil stellt jedoch in vereinzelten Grotten eine größere, an der Hinterwand ausgehobene Nische mit einer halbkreisförmigen Sitzbank (1,5 m lang, $1 \mathrm{~m}$ breit, 0,5 m hoch) dar, die durch einen Einschnitt zweigeteilt ist (Abb. 3). Welchen $Z_{w e c k}$ dieser Bauteil eigentlich zu erfüllen hatte, läßt sich nicht ohne weiteres sagen. Vielleicht handelte es sich um einen Hausaltar. Das ist nicht ausgeschlossen, wohnten im Gebiete doch auch basilianische Mönche. Rechts der «Sitzbank»- der Beschreibung liegt eine ganz bestimmte Grotte zugrunde - weist die ausgebuchtete Wand ein rundes Loch auf, das mit einer kleinen Verebnung über der Grotte in Verbindung steht; ein Pfad führt dorthin. Vielleicht wurden durch dieses «Felsrohr»Oliven in einen Behälter der Grotte eingeschüttet, wie das heute noch in Matmata (Südtunesien) geschieht. Sodann sind neben gewöhnlichen Wandnischen faustgroße Löcher vorhanden, und zwar einen Meter über dem Boden eine Reihe bildend. In sie wurden vermutlich hölzerne Sparrenköpfe gesteckt zum Tragen von Brettchen, auf die bestimmte Gegenstände gelegt werden konnten. Ähnliche Einrichtungen kommen heute noch in den Häusern verschiedener Ortschaften Apuliens vor. Wer diese niedrigen Grotten besucht, dem fallen überdies die aus der Decke oder den Wänden herausgemeißelten Halbringe auf; es handelt sich sicherlich um Aufhängevorrichtungen.

Verschiedene Wohnhöhlen setzen sich aus mehreren, teilweise miteinander verbundenen Räumen zusammen. In den großen Räumen ließ man beim Aushub, der mit der Hacke erfolgte, stützende Pfeiler stehen. Weicheres Material wurde, wie die Musterungen zeigen, mit der Breitseite der Hacke ausgeräumt, härteres Material aber mit der Spitzseite. Ein genaues Studium dieser Wandmuster dürfte dem Fachmann gestatten, Rückschlüsse auf die Art der verwendeten Hacke zu ziehen und damit auf die Entstehungszeit dieser Höhlen. In vielen Räumen ist der Boden im hinteren Abschnitt um 20 
bis $30 \mathrm{~cm}$ erhöht. Auch gibt es im Boden ausgehobene Behälter mit kreisrunder Öffnung (Durchmesser 50-60 cm). Es handelt sich wohl um Bodenspeicher zur Aufbewahrung von Getreide. Solche Speicher sind heute noch in gewissen Gebieten Südtunesiens verbreitet.

Über den Eingängen ist oft ein Loch für den Zutritt von Licht und Luft angebracht und ferner eine aus dem Fels gehauene bogenförmige Rinne, welche das Regenwasser, das über dem darüberliegenden Felshang niederging, aufzufangen und seitlich abzuleiten hatte.

Die Grotten der Gravina della Madonna della Scala sind schon seit langem aufgegeben; niemand aber weiß, warum. Vielleicht des Umstandes wegen, daß von den Talhängen wiederholt Gesteinsblöcke zu Tal fuhren, besonders bei Erdbeben. Wie mir der Stadtarchivar E. Jacovell a af Grund einer Chronik mitteilte, wurde zum Beispiel während eines Erdbebens im Jahre 1696 die breite, in den Fels geschlagene Aufstiegstreppe, die auf der rechten Talseite zu einer Höhlenwohnung emporführte, zerstört; größere Felstrümmer stürzten dabei nieder.

In der Altstadt von Massafra steigen drei Gassen parallel zu den beiden Gravine zur Ebene ab, nämlich die Via Muro, Via La Terra und Via San Nicola. Sie verlaufen in alten, einst von Bächen durchzogenen Gräben. An ihnen liegen Wohnhöhlen. Diese sind aber nicht ohne weiteres zu erkennen, sind ihnen doch aus Mauerwerk bestehende Bauten, wie Küche und Wohnzimmer, vorangestellt. Im Beispiel, das Abb. 4 festhält, geht das $4 \mathrm{~m}$ hohe Wohnzimmer in ein halb so hohes und bereits einen Bestandteil der Wohnhöhle bildendes Schlafzimmer über. Hinter diesem Zimmer folgt noch eine weitere Schlafgrotte. Diese weist einen kleinen Speicher und eine Zisterne auf. Grotten und Vorbauten erscheinen als ein organisches Ganzes. Der gleiche Wohntyp kommt beispielsweise auch in Campo de Criptana der Mancha Spaniens vor. Nach O. Jessen (S. 210) sind dort die Vorbauten wohl erst später der Höhle angegliedert worden. Das dürfte auch für Massafra zutreffen.

An der Via Muro liegen die Wohnhöhlen in zwei bis vier Stockwerken übereinander. $\mathrm{Zu}$ ihnen führen von der Gasse aus geweißte Treppen. Schornsteine verraten die Küchen, die als Vorbauten oder Grotten angelegt sind.

In der Altstadt trifft man auch Höhlen vom Schachttypus, im ganzen wohl über hundert. Sie liegen nahe beisammen und sind bei der Bevölkerung unter der Bezeichnung Vicinanze (Nachbarschaften) bekannt. Ihr Hauptteil ist ein viereckiger, oft quadratischer Schacht oder Hof, welcher 3,5-5 $\mathrm{m}$ tief aus dem Erdboden ausgehoben ist und Seitenlängen von 3-10 m aufweist. An den Hof grenzen, sei es auf einer, zwei oder drei Seiten, niedrige, im allgemeinen zweigeschossige Häuser. Das Ganze erweckt den Eindruck, eine bauliche und zugleich funktionelle Einheit zu sein. Auf der vierten Seite, die an die Gasse angrenzt, ist der Hof zum Schutze für Erwachsene und Kinder von einer Mauerbrüstung eingefaßt.

In den Schacht führt eine aus dem Fels ausgehauene Treppe, die oben entweder von einer Seitenmitte oder einer Ecke ausgeht. Damit sie nicht zu steil wird, nimmt sie unter Umständen schon außerhalb des Schachtraumes ihren Anfang oder verläuft winklig gebogen.

Um den Hof herum gruppieren sich mehrere, meist vier bis acht Grotten. Diese sind 5-10 $\mathrm{m}$ lang, 3-6 $\mathrm{m}$ breit und $3 \mathrm{~m}$ hoch. Sie liegen nur $0,5-1,5 \mathrm{~m}$ unter der Erdoberfläche. Das ist möglich, weil diese aus solidem Kalktuff besteht. Ja diese Grottendecke muß sogar noch zwei- oder dreigeschossige Häuser tragen.

Die Grotten der Vicinanze haben verschiedene Aufgaben zu erfüllen. Die einen dienen als Weinkeller, die andern als Schuppen für Hausrat, Werkzeug, Gartengeräte usw., als Holz- und Strohmagazine oder als Ställe für Kühe und Esel. Vereinzelte sind in den letzten Jahren zu sauberaussehenden, geweißten Werkstätten, zum Beispiel für Schreiner oder Maler, umgewandelt worden. Diese Grotten werden aber nicht bewohnt. Doch dürfte das früher der Fall gewesen sein, wie vereinzelt in ihnen vorkommende 
Kochstellen zeigen. Manchmal haben als solche auch unter der Treppe ausgehobene $\mathrm{Ni}$ schen gedient. Laut Auskunft von seiten älterer Leute wurden in diesen Grotten früher landwirtschaftliche Hilfskräfte einquartiert.

Das Regenwasser, das über dem Hof niedergeht, fließt einer dort gelegenen Zisterne zu. Vereinzelte Grotten sind selber mit Zisternen ausgestattet. Ihnen wird das Regenwasser, das zum Beispiel über der angrenzenden Gasse fällt, zugeleitet. Dieses Wasser dient zum Tränken des Viehs und als Brauchwasser.

Was Massafra aber eine ganz besondere Note verleiht, sind die zahlreichen unterirdischen Krypten. Allein in seinem näheren Umkreis zählt man 26, im ganzen Gebiet 50. Sie stammen, wie aus den vielen, leider größtenteils arg zerfallenen Fresken, biblische Szenen darstellend, hervorgeht, aus verschiedenen Epochen. Massafra muß einst ein hervorragendes religiöses Zentrum gewesen sein. Inschriften, Bilddarstellungen und Altarformen weisen auf eine seit dem 6. Jahrhundert erfolgende griechisch-byzantinische Einwanderung hin. Diese Schluchttäler waren nach E. JACovelli ${ }^{2}$ (S.7) zur Aufnahme dieser orientalischen Bevölkerungen die geeigneten Stellen. Damals wohl entstanden schon für Mönche und Weltliche Wohnhöhlen. Die einen Krypten gehören zum Hangtypus, wie zum Beispiel die Krypten San Marco und La Candelora, die sich in den Hängen der Gravina di San Marco befinden, und die andern, wenn in ebenem Gelände erstellt, zum Schachttypus, wie die heute unter dem Erdgeschoß des Spitals gelegene Krypta Sant'Antonio Abbate.

Vom Menschen bewohnte Höhlen treten noch in anderen Gebieten Süditaliens auf, so nach C. LAnza und G. CAPPA ${ }^{3}$ in der am Südabhang der Halbinsel Gargano gelegenen Stadt Monte Sant'Angelo. Auf Höhlen gleicher Art stieß ich in Peschici, einer Ortschaft an der Nordküste der gleichen Halbinsel. Nur werden sie dort heute, von ein oder zwei Ausnahmen abgesehen, nicht mehr bewohnt, sondern als Ställe und Schuppen benützt.

\section{HABITATIONS TROGLODITIQUES EN ITALIE MÉRIDIONALE}

Actuellement encore, un nombre restreint de la populaiton de l'Italie méridionale habite des demeures trogloditiques, ainsi dans les villes de Matera (Basilicata), Massafra (Puglie) et Monte Sant'Angelo (presque-île de Gargano). A Matera cependant, un vaste mouvement de déplacement prend son cours: la population quitte les grottes pour s'installer dans des maisons. De nouveaux quartiers ont surgi et même de nouveaux villages alentours. A Massafra, les demeures trogloditiques encore habitées de la vieille ville valent, certes, la peine d'être vues, mais surtout les très anciennes grottes de la gorge Gravina della Madonna della Scala et celles, nombreuses, représentant d'anciennes cryptes, sont d'un intérêt tout spécial.

\section{NEUTRALES LAND - IN NORDARABIEN}

\section{Hans Boesch}

Dieser Artikel wird im Zusammenhang mit der dieser Nummer der Geocraphica Helvetica beigegebenen Karte "Naher Osten", 1:5000000, des Verlages Kümmerly + Frey, Bern, publiziert

An der Nordgrenze Saudi-Arabiens verzeichnen die meisten unserer Karten zwei sogenannte Neutrale Zonen. Die eine (westliche) liegt eingeschaltet zwischen SaudiArabien und Irak, die andere (östliche) zwischen dem Scheichtum Kuwait und SaudiArabien. Da es sich hier um zwei ausgesprochene Kuriosa der Grenzgeographie handelt, über die zudem im allgemeinen Genaueres nicht bekannt ist, seien sie im Hinblick auf ihre aktuelle Bedeutung im folgenden kurz besprochen.

2 E. Jacovelli: Gli affreschi bizantini di Massafra. Massafra 1960, 46 S.

3 C. Lanza und G. Cappa: Indagine preliminare sulle abitazioni trogloditiche a Monte Sant' Angelo. Bollettino della Società Geografica Italiana. Roma 1962. S. 193-203. 